\title{
Melanization in living organisms: a perspective of species evolution
}

\author{
Christopher J Vavricka ${ }^{1 凶}$, Bruce M. Christensen², Jianyong Li $^{3}$ \\ ${ }^{1}$ CAS Key Laboratory of Pathogenic Microbiology and Immunology, Institute of Microbiology, Chinese Academy of Sciences \\ (CASPMI), Beijing 100101, China \\ 2 Department of Pathobiology, University of Wisconsin-Madison, Madison, WI 53706, USA \\ 3 Department of Biochemistry, Virginia Tech, Blacksburg, VA 24061, USA \\ $\triangle$ Correspondence: chris@im.ac.cn
}

Received August 30, 2010 Accepted September 8, 2010

\begin{abstract}
Eumelanin is a heteropolymer that is generally composed of hydroxylated indole residues and plays diverse protective functions in various species. Melanin is derived from the amino acid tyrosine and production of melanin is a highly complex oxidative process with a number of steps that can either proceed enzymatically or non-enzymatically. Although melanin plays important protective roles in many species, during melanization, particularly in steps that can proceed non-enzymatically, many toxic intermediates are produced, including semiquinones, dopaquinone, indole-quinones and moreover, the production of many reactive oxygen species. To mitigate the production of reactive species, a number of proteins that regulate the biochemical process of melanization have evolved in various living species, which is closely related to adaptation and physiological requirements. In this communication, we discuss differences between non-enzymatic and enzymatic processes of melanization and the enzymatic regulation of melanization in difference species with an emphasis on differences between mammals and insects. Comparison between melanization and insect sclerotization is also emphasized which raises some interesting questions about the current models of these pathways.
\end{abstract}

KEYWORDS melanization, melanogenesis, sclerotization, tanning, dopa, tyrosinase, dopachrome tautomerase

\section{NON-ENZYMATIC PRODUCTION OF MELANIN}

It is well known that incubation of L-dopa (D-dopa as well) in neutral buffer with the presence of molecular oxygen leads to the production of melanin. The reaction must be initiated by oxidation of dopa by molecular oxygen since essentially no melanin is produced if oxygen is completely eliminated from the L-dopa solution. Oxygen cannot accept two electrons at the same time. As a result, oxidation of $\mathrm{L}$-dopa by $\mathrm{O}_{2}$ produces a dopa-semiquinone radical and superoxide (Fig. 1) (Nappi and Vass, 1993; Nappi et al., 1995). Two molecules of semiquinone might undergo dismutation, leading to the production of one molecule of dopaquinone and one molecule of L-dopa (Fig. 1). The dopa-semiquinone might be oxidized by superoxide, resulting in the formation of dopaquinone and hydrogen peroxide, respectively (Fig. 1).

Under neutral or basic $\mathrm{pH}$ conditions, dopaquinone undergoes rapid intramolecular cyclization, which produces leukodopachrome (Fig. 2) (Cánovas et al., 1982). Leukodopachrome, however, is much more reactive than L-dopa and in-turn is oxidized more easily by $\mathrm{O}_{2}$ or by other reactive species, including dopa-semiquinone, superoxide, hydrogen peroxide, and so forth. This leads to the production of dopachrome as a relatively stable intermediate along the melanization pathway (Fig. 2). Under relatively acidic conditions, however, the side chain amino group is protonated, which prevents intramolcular nucleophilic cyclization through the amino group. Under such a condition, the oxygen of the side chain carboxyl group undergoes similar nucleophilic addition, leading to the production of a lactone intermediate that also is unstable and undergoes hydrolysis to 6-hydroxylated quinone species (Li et al., 1996). It is clear that during the non-enzymatic process of L-DOPA to dopachrome, numerous reactive and toxic species, including superoxide and semiquinone, are produced.

Dopachrome (DC), once formed, undergoes structural rearrangement to form 5,6-dihydroxyindole (DHI) under 


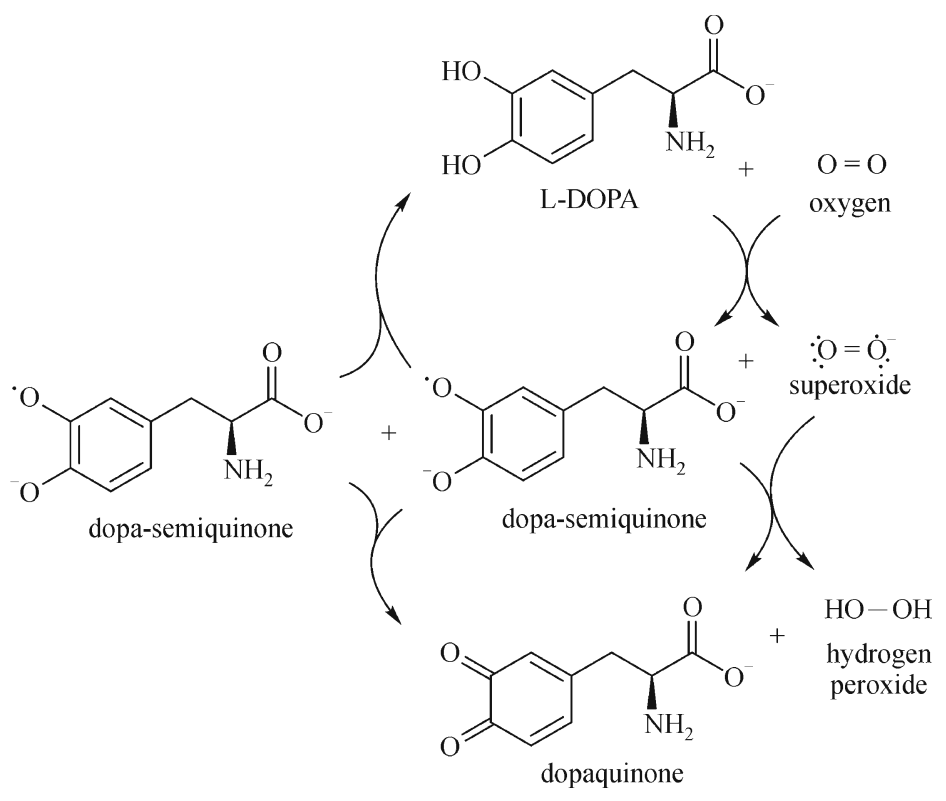

Figure 1. Proposed reactions for the non-enzymatic formation of dopaquinone from L-dopa. Oxidation of L-dopa by $\mathrm{O}_{2}$ produces dopa-semiquinone and superoxide which may react to form dopaquinone and hydrogen peroxide. Two molecules of dopasemiquinone may also react with eachother to form one molecule of dopaquinone and one molecule of L-dopa.<smiles>N[C@@H](Cc1ccc(O)c(O)c1)C(=O)[O-]</smiles>

L-DOPA<smiles>CC(C)C1Nc2cc(O)c(O)cc2CC1C(=O)[O-]</smiles><smiles>O=C1C=C2CC(C(=O)O)N=C2C=C1O</smiles>
dopachrome $\mathrm{CO}_{2} \&$ decarboxylative<smiles>Oc1cc2cc[nH]c2cc1O</smiles>

5, 6-dihydroxyindole (DHI)

Figure 2. Overview of the general reactions for non-enzymatic formation of melanin. Amino and carboxyl groups are represented in their deprotonated forms as this process occurs more easily under basic conditions or in the presence of neutral or basic buffer.

neutral conditions ( $\mathrm{pH} 6.0-7.0)$ and the rate of dopachrome to $\mathrm{DHI}$ depends on the concentration of neutral buffer (Fig. 2 and 3). Overall, the rate of dopachrome to form $\mathrm{DHI}$ is relatively slow in the presence of low concentrations of buffer, and the rate of $\mathrm{DHI}$ formation increases as buffer concentrations increases (Fig. 3).
Dopachrome to DHICA conversion can be promoted by the presence of divalent metal cations (Fig. 4A) (Palumbo et al., 1987, 1991b). Moreover, pH and different buffer species have major impacts on the subsequent chemical reactions of dopachrome. At slightly basic conditions, such as phosphate buffer at $\mathrm{pH} 7.5-8.0$, some DC is converted to 5,6- 


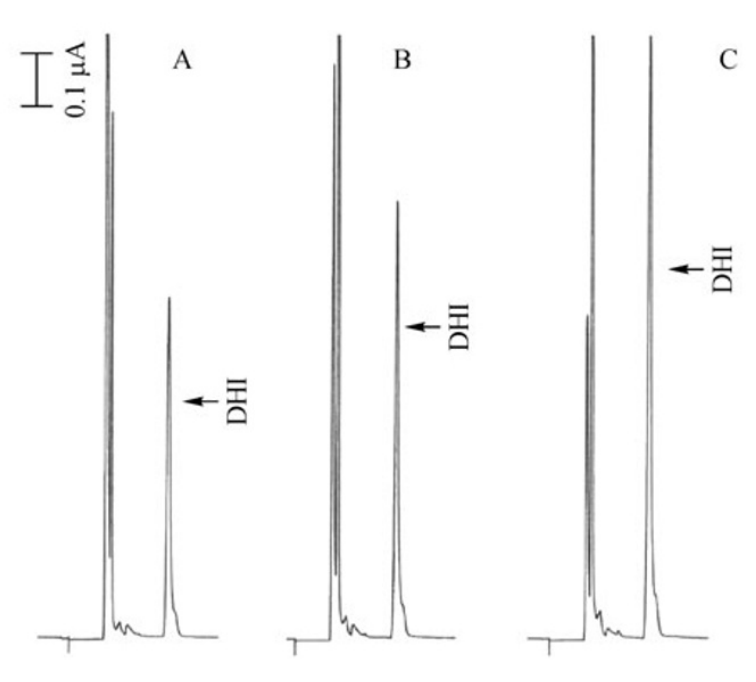

Figure 3. Effect of buffer concentrations on the conversion of dopachrome to DHI. Dopachrome solution (4 mM) was prepared by mixing an equal volume of $4 \mathrm{mM}$ L-dopa prepared in distilled water and an equal volume of $8 \mathrm{mM}$ sodium periodate prepared in $5 \mathrm{mM}$ phosphate buffer. The freshly prepared dopachrome solution was then mixed with an equal volume of different concentrations of phosphate buffer (pH 7.0) containing $5 \mathrm{mM}$ ascorbate (to stabilize $\mathrm{DHI}$ and DHICA formed in the reaction mixtures). The dopachrome and buffer mixtures were incubated for $10 \mathrm{~min}$ and then analyzed by HPLC with electrochemical detection (HPLC-ED). Chromatograms $(A),(B)$ and $(C)$ illustrate $\mathrm{DHI}$ formed in dopachrome solution in the presence of $125 \mathrm{mM}, 250 \mathrm{mM}$ and $500 \mathrm{mM}$ phosphate buffer ( $\mathrm{pH} 7.0)$, respectively.

dihydroxyindole-2-carboxylic acid (DHICA), but decarboxylative structural rearrangement to $\mathrm{DHI}$ remains a major pathway. When commonly used HEPES buffer at $\mathrm{pH} 7.5$ is mixed into dopachrome solution, production of DHICA becomes apparent (Fig. 4B) and rate of DHICA formation almost matches up with that of $\mathrm{DHI}$ at $\mathrm{pH} 9.4$ (Fig. 4C). However, when Tris buffer at $\mathrm{pH} 8.5$ is mixed into dopachrome solution, conversion of dopachrome to DHICA is diminished and production of $\mathrm{DH}$ from dopachrome remains a major route (Fig. 4D). These data suggest that while relatively basic $\mathrm{pH}$ promotes the conversion of dopachrome to DHICA in general, the chemical and physical characteristics of the buffer species also greatly affect the chemical reactions of dopachrome and it should be interesting to elucidate the specific chemical mechanisms of dopachrome chemical reactions under different conditions.

$\mathrm{DHI}$ and DHICA can be further oxidized to their respective indole-quinones in a similar manner to catechol oxidation (Fig. 5) (Prota, 1992). During the oxidation of catechols and dihydroxyindoles to their respective quinones, the hydroxyl groups must undergo a loss of 2 protons. Therefore, $\mathrm{pH}$ also plays a critical role in the rate of quinone formation ( $\mathrm{Li}$ and

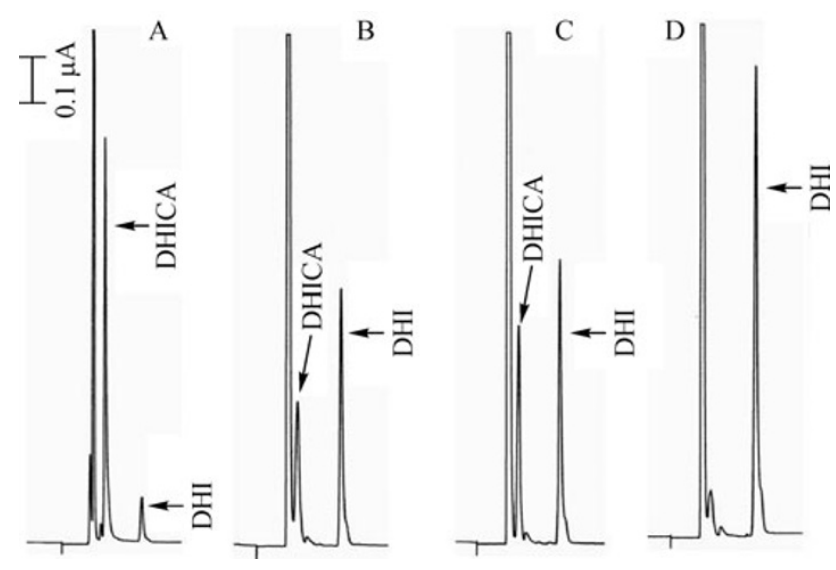

Figure 4. Effect of metal ions, $\mathrm{pH}$ and buffer species on the subsequent chemical reactions of DHI and DHICA. Dopachrome solution ( $4 \mathrm{mM}$ ) was prepared by mixing an equal volume of $4 \mathrm{mM}$ L-dopa prepared in distilled water and an equal volume of $8 \mathrm{mM}$ sodium periodate prepared in $5 \mathrm{mM}$ phosphate buffer. The freshly prepared dopachrome solution was then mixed with an equal volume of $2 \mathrm{mM}$ copper sulfate prepared in $5 \mathrm{mM}$ phosphate buffer ( $\mathrm{pH} 7.0), 200 \mathrm{mM}$ HEPES buffer ( $\mathrm{pH} 7.5), 200 \mathrm{mM}$ Tris buffer ( $\mathrm{pH} 8.5$ ) or $200 \mathrm{mM} \mathrm{CAPS}$ buffer ( $\mathrm{pH}$ 9.4) containing $5 \mathrm{mM}$ ascorbate (to stabilize $\mathrm{DHI}$ and DHICA formed in the reaction mixtures). The dopachrome and copper sulfate or buffer mixtures were incubated for 5-10 min and then analyzed by HPLC with electrochemical detection (HPLC-ED). Chromatogram (A) shows the accumulation of a high concentration of DHICA and low concentration of $\mathrm{DHI}$ in dopachrome solution in the presence of copper sulfate. Chromatograms (B), (C) and (D) illustrate the relative amounts of DHICA and $\mathrm{DHI}$ formed in the reaction mixtures in the presence of HEPES ( $\mathrm{pH} \mathrm{7.5),} \mathrm{CAPS}(\mathrm{pH} \mathrm{9.4)}$ and Tris buffer ( $\mathrm{pH}$ 8.5), respectively. The final concentration of copper was $1 \mathrm{mM}$ and the final concentration of buffer for HEPES, CAPS and Tris was $100 \mathrm{mM}$.

Christensen, 1994).

$\mathrm{DHI}$, once formed, can be oxidized to DHI-quinone by $\mathrm{O}_{2}$ and reactive oxygen species, semi-dopaquinone, dopaquinone, etc. In contrast, DHICA is not oxidized easily by $\mathrm{O}_{2}$. In the conversion of dopa to DHI-quinone, the molecule has lost 6 -electrons. After each round of oxidation, the molecule undergoes structural rearrangement for stabilization, and this actually results in the formation of a more reactive compound. For example, both leukodopachrome and $\mathrm{DHI}$ are more easily oxidized by $\mathrm{O}_{2}$ than L-dopa. Therefore, while melanin polymers are beneficial to many species, including humans, the process of its production leads to the formation of many reactive, toxic species. This likely explains the evolvement of other regulatory proteins along the melanization pathway in different species. Finally, DHI- and DHICA-quinone, formed in the reaction mixture, cannot undergo further structural rearrangement alone for stabilization, and polymerization of the reactive species results in the production of melanin 


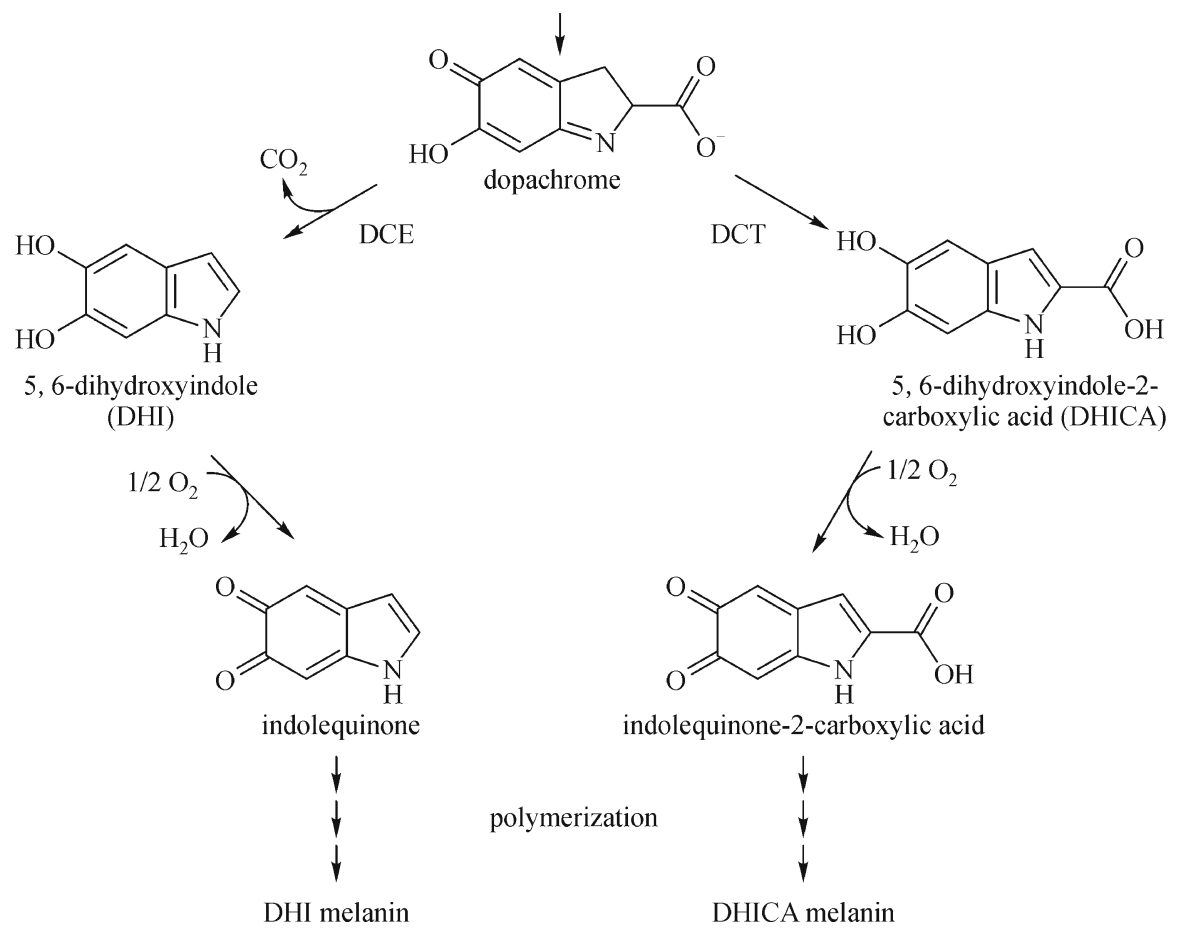

Figure 5. Direct enzymatic regulation of melanization. The mammalian enzyme DCT and insect enzyme DCE promote formation of DHICA melanin and DHI melanin, respectively.

polymers (Fig. 2 and 5).

\section{MELANIZATION IN PRIMITIVE SPECIES}

Although the oxidation of L-dopa to dopaquinone may occur non-enzymatically under aerobic conditions, many bacteria and fungi contain tyrosinase to accelerate the same reaction. Production of dopaquinone may also be catalyzed by hemocyanin, laccase or catechol oxidase; however tyrosinase is well known to be specifically involved in melanin biosynthesis. Tyrosinase is a rather unique enzyme. It catalyzes the hydroxylation of tyrosine and other monophenols to catechols and oxidizes diphenolic compounds to their o-quinones (Matoba et al., 2006). Accordingly, tyrosinase behaves as both a monooxygenase (hydroxylase) and a dioxygenase. Moreover, it also catalyzes the oxidation of $\mathrm{DHI}$ to its corresponding indole-quinones along the eumelanin synthetic pathways (Olivares et al., 2001). Tyrosinase catalyzes two-electron oxidation of precursors and intermediates along the melanization pathway and transfers the two electrons to oxygen: i.e., reducing oxygen directly to water. As a result, this enzyme enormously reduces the production of reactive oxygen species and semi-quinone radicals during the melanization process and therefore is critically important for the production of melanin in living species.

It has generally been accepted that tyrosine is the initial precursor and hydroxylation of L-tyrosine to L-dopa is the limiting step in the melanization pathway in bacteria and fungi. This likely is true because the rate of tyrosine hydroxylation by tyrosinase is at least one order of magnitude lower than its activity to dopa (Jara et al., 1988). It should be noted that the individual monophenolase and diphenolase activities of tyrosinase may vary within different species and that there is some difficulty in separating these two tyrosinase mediated activities (Hernández-Romero et al., 2006). Tyrosine can be hydroxylated to L-dopa by tyrosine hydroxylase, but such an enzyme does not seem to be present in fungi. Although a putative tyrosine hydroxylase seems to be present in some bacteria, the actual function of the protein in tyrosine hydroxylation has not been clearly established and there has been no discussion regarding its involvement in melanin synthesis in bacteria. Another rate limiting step in the melanization pathway is the conversion of dopachrome to $\mathrm{DHI}$ (although it was not emphasized or considered to be a rate limiting step in many earlier reports). After the production of dopaquinone, subsequent melanization reactions may proceed spontaneously under oxidizing conditions. This likely is the case for melanization in bacteria because they do not posses other known enzymes to manipulate this process aside from tyrosinase. As discussed for non-enzymatic melanization, the rate of dopachrome to $\mathrm{DHI}$ is affected by $\mathrm{pH}$, buffer concentrations and the chemical/physical characteristics of the buffers (Fig. 3 and 4). However, during the course of eukaryote evolution, this branch pathway has been extensively fined-tuned in many other species, espe- 
cially in insects and mammals.

Most melanin arising from tyrosine in non-mammalian species is thought to proceed through the decarboxylative pathway leading to formation of the black, insoluble $\mathrm{DHI}$ melanin (Prota, 1992) (Fig. 5). DHI is easily oxidized to its oquinone that polymerizes to form melanin. No additional enzymes have yet been discovered outside of the animal kingdom; however it is interesting to note that yeast often contain many homologous tyrosinase sequences.

\section{ENZYMATIC REGULATION OF MAMMALIAN MELANOGENESIS}

Melanogenesis is an important physiological event in humans, which takes place in melanocytes at the skin epidermis layer. Melanocytes originate in the neural crest and migrate to the basal layer of the epidermis and the hair matrices. Under physiological conditions, melanin synthesis in melanocytes is restricted to the melanosomes, a subcellular organelle that is formed in a process similar to lysosome formation. Like the lysosome, the melanosome contains a low $\mathrm{pH}$ environment, which is useful for the control of melanogenesis, since many of the oxidation reactions occur more slowly under acidic conditions.

Melanosome development is commonly divided into four stages based on structural formation and changes with stage I corresponding to the early matrix organization, stage I| displaying a well organized matrix (without melanin), stage III containing some deposited melanin and stage IV being filled with melanin. Melanogenesis in melanocytes and melanosome transfer from melanocytes to keratinocytes are induced by UV radiation and keratinocyte growth factor (Cardinali et al., 2008). Mature melanosomes filled with melanin polymers are transferred to the neighboring keratinocytes in the epidermis and protect DNA against UV damage. Genetic defects and environment factors (especially the overexposure to ultraviolet radiation) may lead to the development of melanoma from melanocytes. Therefore, the cascades of the genetic regulatory pathways of mammalian melanogenesis and the pathological consequences resulting from the disorder or interruption of the normal developmental process have been attracting considerable attention. This has led to major progress in understanding of the physiology and pathology of mammalian melanogenesis. There have been many primary research articles and review articles dealing with the genetic regulation of the mammalian melanogenesis pathway (Hearing, 1999; Cheli et al., 2009). To compare melanogenesis in different species, our discussion regarding mammalian melanogenesis will be focused on proteins that are directly involved in enzymatic regulation of melanin synthesis.

For many years tyrosinase, capable of catalyzing the hydroxylation of tyrosine and oxidation of L-dopa and $\mathrm{DHI}$ in the melanization pathway, has been considered the only enzyme involved in the mammalian melanin biosynthesis. Since the discovery of tyrosinase in melanization, this enzyme has been one of the most extensively studied proteins in living organisms. Subsequently, it has become clear that several other proteins, including phenylalanine hydroxylase, tyrosine hydroxylase, tyrosinase-related protein-1 (TRP-1) and tyrosinase-related protein-2 (TRP-2), are also directly involved in mammalian melanogenesis. Although tyrosinase is able to catalyze the hydroxylation of tyrosine to L-dopa, it is much less efficient in mediating the reaction. It has now generally been accepted that in humans, L-dopa required for the synthesis of melanin polymers is primarily derived from phenylalanine by phenylalanine hydroxylase- and tyrosine hydroxylase-mediated reactions (Marles et al., 2003). Consequently, the primary initial precursor in human melanogenesis should be phenylalanine.

Perhaps the major difference between melanogenesis in mammals and that of non-mammalian species is the dopachrome to DHICA branch pathway. For this reason we use "melanization" as a term that is more specific for the predominantly spontaneous decarboxylative melanin pathway that occurs chemically or in insects, and "melanogenesis" as a more specific term for the highly regulated mammalian DHICA related pathway. It was observed that a factor accelerating the conversion of dopachrome to downstream intermediates is present in mammals (Körner and Pawelek, 1980; Hearing et al., 1982) and was named dopachrome conversion factor (Korner and Gettins, 1985; Lamoreux et al., 1986) or dopachrome oxidoreductase (Barber et al., 1984). The product resulting from dopachromedopachrome oxidoreductase reaction was subsequently identified to be DHICA (Leonard et al., 1988). Based on its catalytic function, the enzyme has also been named dopachrome isomerase (Pawelek, 1990) and dopachrome tautomerase (DCT) (Aroca et al., 1990). These earlier studies established the presence of an additional enzyme that catalyzes the conversion of dopachrome to DHICA in the melanogenesis pathway. After the presence of DCT in mammals was established, there were a number of reports dealing with the purification and biochemical characterization of mammalian DCT from melanocytes or melanomas (Aroca et al., 1991; Palumbo et al., 1991a; Townsend et al., 1992). A cDNA corresponding to DCT was first isolated from mouse and genetic mapping of mouse DCT localized the gene on chromosome 14 at a pigmentation locus called slaty (Jackson et al., 1992). Comparison of DCT from wild-type with that of slaty mice revealed a single amino acid difference and DCT from the slaty mice displayed greatly reduced activity (Jackson et al., 1992).

In many species, $\mathrm{DHI}$ is the primary intermediate that is one step away from melanin polymers, and formation of melanin polymers seems to be more rapid with DHI than with DHICA. This leads to an essential question regarding the driving force for the evolution of an enzyme that redirects dopachrome to 
DHICA in mammalian melanogenesis. When DHI and DHICA standards are dissolved in neutral phosphate buffer in test tubes, more precipitated melanin is observed in a DHI solution as compared with a DHICA solution during a short incubation period. In the absence of other oxidizing agents, oxygen apparently is the agent causing $\mathrm{DHI}$ oxidation in neutral buffer, which leads to the formation reactive oxygen species and semi-quinone radicals that likely are highly toxic to melanocytes. Indeed, melanoma and other cells exposed to sub-millimolar concentrations of DHI are lysed or killed in just a few hours (Pawelek and Lerner, 1978). Quantum chemical studies of DHI and DHICA confirm that DHICA is considerably more stable than $\mathrm{DHI}$ based on the presence of unpaired electrons in DHI (Powell, 2005). Melanin produced from DHICA exhibits a potent hydroxyl radical-scavenging activity, but melanin produced from $\mathrm{DHI}$ does not and the ability of skin photoprotection in DCT knockout mice against ultraviolet radiation are greatly compromised (Jiang et al., 2010). It is conceivable that DHICA is less vulnerable to nonenzymatic oxidation by molecular oxygen and that melanin derived from DHICA is able to better quench radical species. Obviously the presence of DCT can greatly reduce oxidative stress by reducing $\mathrm{DHI}$ formation and possibly enhance the production of melanin with better capacity to scavenge reactive radical species during melanogenesis. This in-turn may explain the evolvement of DCT in mammals. On the other hand, the presence of DCT also benefits melanoma because the cancer cells become resistant to common chemotherapy and radiation (Pak et al., 2004). The overexpression of DCT in WM35 melanoma cells and HEK epithelial cells further reduces their sensitivity to oxidative stress (Michard et al., 2008a, b). Furthermore, DCT derived peptides have been extensively studied as melanoma antigens presented by MHC (Wang et al., 1996).

Based on available data about DCT, it is reasonable to propose that DCT plays an important role in protecting melanocytes from toxic effect of $\mathrm{DHI}$ and other oxidative stress. However, the underlying mechanism of its protective function is far from clear; and although the catalytic function of DCT in the isomerization/tautomerization of dopachrome to DHICA has been clearly established, the chemical mechanism and structural basis of its substrate binding and catalysis are unclear. A major obstacle for a comprehensive understanding of mammalian DCT has been the amount of protein available for study. There is a high level of difficulty obtaining the highly processed and glycoslated proteins. Currently there are no crystal structures available for mammalian tyrosinase, DCT or TRP-1. Our recent study indicates that it is possible to obtain a relatively large quantity of mammalian DCT through its expression in insect cells (Vavricka et al., 2010), which makes it possible to obtain adequate amounts of enzyme for critical structural characterization of mammalian DCT.

\section{INSECT MELANIZATION}

Most insects rely heavily upon melanization for the hardening of their cuticle and have also evolved some interesting enzymes for the metabolism of L-dopa. It is generally accepted that the highly reactive intermediates of melanization contribute to the crosslinking of cuticular proteins, which results in the formation of a protective cuticle. In insects phenoloxidase $(P O)$ is the equivalent to tyrosinase in other species. Insect PO, as tyrosinase, can catalyze the hydroxylation of tyrosine to dopa, oxidation of dopa to its o-quinone, and oxidation of $\mathrm{DHI}$ to its indole-quinone along the melanization pathway. However, insect POs share little sequence homology to tyrosinase. Moreover, unlike the presence of single tyrosinase gene in mammals and other organisms, insects usually contain multiple PO genes and they are expressed as proenzymes commonly named prophenoloxidase (proPO). There has been a great amount of research dealing with the function and activation of insect proPO (Li et al., 2005), and this will not be further discussed and elaborated in this communication.

Similar to the melanization pathway in other species, PO used to be considered the only protein catalyzing insect melanization reactions. Compared to tyrosinase, insect PO seems more efficient in catalyzing the hydroxylation of tyrosine or other monophenolic compounds (personal experience). In a reaction mixture of L-dopa and phenoloxidase, there is a rapid oxidation of L-dopa to dopaquinone, intramolecular nucleophilic cyclization of dopaquinone to leukodopachrome and oxidation of leukodopachrome to dopachrome. This is evident by the accumulation of dopachrome that appears bright red at neutral $\mathrm{pH}$. Because the direct enzymatic product of dopaquinone does not accumulate, the reaction essentially goes to completion and all of the L-dopa molecules are converted to dopachrome and the solution remains red for hours before turning black (melanin accumulation). However, when a crude protein sample, extracted from insects, such as from mosquito larvae or pupae, is used as an enzyme source, the reaction mixture turns black very rapidly with hardly noticeable bright red color at any time during the reaction. This suggests that, in addition to $\mathrm{PO}$, there is at least one more protein that is able to promote insect melanization. A similar protein, which accelerates the melanization process, has also been reported in other insect species (Aso et al., 1984). Based on its function, a mosquito enzyme for melanization was discovered and named dopachrome conversion enzyme (DCE) (Li et al., 1994, 2007).

A primary sequence of DCE was first determined through molecular cloning based on partial sequences of purified mosquito DCE (Johnson et al., 2001) and a BLAST search reveals that DCE shares no noticeable sequence similarity to proteins from any other organisms, except with proteins from the insect yellow gene family. The identification of mosquito 
DCE subsequently led to the identification of Drosophila yellow $f$ and yellow $f 2$ as DCE (Han et al., 2002). Since then these sequences have been used as references to annotate counterpart genes from other insect species. Now it has become clear that DCE that catalyzes rapid conversion of dopachrome to $\mathrm{DHI}$ is present in many insect species and this protein increases the rate of melanization in insects.

Insect DCE is somewhat comparable with mammalian DCT because they use the same substrate and are involved in the same melanization pathway (although their products are different). As a result, it seems reasonable to consider insect DCE the counterpart of mammalian DCT, but surprisingly, insect DCE shares no sequence similarity with mammalian DCT. DHI is highly reactive, while DHICA is relative stable. Moreover, melanin polymers produced from DHICA seem to be more protective to human skin against UV irradiation (Jiang et al., 2010). This leads to a critical question as to why DCE, instead of a DCT type of protein, has evolved in insects.

\section{MELANIZATION AND SCLEROTIZATION IN INSECT CUTICLE TANNING/HARDENING}

Melanization is intimately related to the formation of hardy, rigid structures (such as the mandible) in many insect species. However, although not explicitly stated, melanization often is not considered a primary biochemical process in the formation of rigid, highly protective cuticle in insects. Cuticle, commonly named exoskeleton, is an extracellular layer covering the outer surface of insects. In insects, tanning and/or sclerotization have been frequently used to describe the biochemical process leading the formation of hardened insect cuticle. It has generally been considered that during tanning/sclerotization process, phenolic compounds, specifically $\mathrm{N}$-acetyldopamine (NADA) and $\mathrm{N}$ - $\beta$-alanyldopamine (NBAD), are oxidatively incorporated into the cuticular matrix, resulting in increased stiffness of the material, decreased water content, and increased resistance toward enzymatic degradation (Andersen, 2010). In many insects, their tanned or sclerotized cuticle is often darkened considerably as compared to their newly formed cuticle. This suggests that melanization should be one of overall biochemical processes leading to the formation of sclerotized cuticle, but there has been essentially no clear discussion regarding the specific contribution of melanization in the formation of tanned/ sclerotized cuticle.

NADA and NBAD have been considered the primary protein crosslinking precursors. Oxidation of these two compounds produces their respective o-quinones that are highly reactive and that directly crosslink cuticle proteins or convert to quinone methide and then crosslink cuticle proteins (Karlson and Sekeris, 1962; Hopkins et al., 1982). NADA is produced from dopamine and acetyl CoA by dopamine $\mathrm{N}$ acetyltransferase and NBAD is produced from dopamine and $\beta$-alanine by NBAD synthetase. Both compounds have been detected in some insect species and enzymes responsible for their synthesis have also been detected in a number of insects (Karlson and Sekeris, 1962; Hopkins et al., 1982). It is reasonable to predict that cuticle protein crosslinking mediated by reactive species from NADA and NBDA oxidations is the major biochemical event responsible for the formation of rigid, protective cuticle in perhaps many insect species.

For some insect species, however, it also is possible that NADA-quinone or NBDA-quinone mediated protein crosslinking might not be the major biochemical event in the formation of a protective cuticle. For example, L-dopa and dopamine often can be detected from mosquito supernatant extracts, but we have repetitively tried to detect NADA in different developmental stages of Ae. aegypti mosquitoes without success. Genome sequences of three mosquito species are available in the database and all enzymes (including tyrosine hydroxylase, L-dopa decarboxylase, dopamine $\mathrm{N}$-acetyltransferases and NBDA synthetase) required for the synthesis of NADA are present in their genomes. Our recent data (unpublished) determined that there are 9 putative aryalkylamine acetyltransferases in Ae. aegypti and functional expression determined that five of them are capable of catalyzing production of NADA during in vitro assays in the presence of dopamine and Acetyl CoA. Feeding of adult females with $2 \mathrm{mM}$ dopamine resulted in the detection of high concentrations of dopamine in mosquito supernatant extract several hours after feeding, but there was no apparent accumulation of NADA in the extract. On the other hand, cuticle melanization is always a prominent phenomenon during cuticle hardening in mosquitoes. One might argue that while NADA-quinone or NBDA-quinone mediated protein crosslinking (sclerotization) is the major biochemical event in cuticle tanning/hardening in many insect species, cuticular melanization could be a primary event in the formation of a protective cuticle in some insect species as well.

Insect sclerotization primarily implies cuticle protein crosslinking reactions. Likewise, a sclerotized cuticle often is equivalent to a rigid, protective cuticle. Based on the fact that cuticle hardening is frequently accompanied with cuticle melanization in many insect species, it is reasonable to propose that melanization is one of the major biochemical events involved in insect cuticle hardening or sclerotization. However, DHI-quinone, the key intermediate in insect melanization, is highly reactive and polymerizes to form heterogeneous polymers once formed. This seemed to have led to the belief that melanization is not related to protein crosslinking. This also might explain a lack of critical discussion and comparison (or hesitation to discuss) between NADA-quinone or NBDA-quinone mediated protein crosslinking or sclerotization reactions and melanization reaction in overall cuticle hardening. 
During L-dopa oxidation by tyrosinase in vitro, some unrelated proteins, if incorporated into the reaction mixtures, become associated with melanin polymers, suggesting that $\mathrm{DHI}$-quinone or its short polymers may crosslink with proteins. In cuticle with densely packed proteins, the possibility of newly formed DHI-quinone or its short polymers to crosslink through nucleophilic groups on proteins likely is much greater than in solution. In mosquito cuticle (perhaps cuticle of many other insect as well), melanin polymers seem to be tightly associated with proteinaceous materials. One might wonder what forces or bonds hold melanin polymers together with other cuticle components. We speculate that $\mathrm{DHI}$-quinone and its polymerized hetero-oligomers may be able to crosslink with cuticle proteins as well. If cuticle sclerotization refers to biochemical events leading to the formation of sclerotized (hardened) cuticle, perhaps cuticle melanization should be considered one of the primary events of sclerotization. For some insect species, such as mosquitoes, cuticle melanization may play more important role than NADA-quinone or NBDA-quinone mediated protein crosslinking for the formation of a protective cuticle. To achieve a comprehensive understanding of melanization in cuticle tanning/hardening and its relation to the commonly considered typical cuticle sclerotization reactions, it is necessary to establish whether $\mathrm{DHI}$-quinone or its short oligomers can crosslink protein and how melanin polymers interconnect with cuticle proteins in the densely packed insect cuticle.

\section{INTRAMOLECULAR CYCLIZATION: A KEY CONCEPT IN MELANIN PATHWAYS}

A fundamental difference between insects and mammals relating to melanin biosynthesis is the use of dopamine conjugates $\mathrm{N}$-acetyl-dopamine (NADA) and $\mathrm{N}$ - $\beta$-alanyldopamine (NBAD) as crosslinking precursors to promote the hardening of the insect cuticle that accompanies cuticle tanning and melanization (Karlson and Sekeris, 1962; Hopkins et al., 1982). As explained above, dopaquinone and dopaminequinone easily undergo intramolecular cyclization leading to the formation of dopachrome and dopaminechrome, respectively (Fig. 6). The nucleophilic amino group within a dopachrome or dopaminequinone molecule is already positioned close to electron deficient quinone ring and therefore is more likely to react than other molecules diffusing in solution. The intramolecular cyclization process allows for the generation of dihydroxylated indole residues as primary melanin residues, rather than dihydroxyphenols. Indoles, which are able to absorb more UV light than their corresponding dihydroxyphenols, are therefore a better choice for photoprotective melanin.

Both NADA and NBAD contain modified amide nitrogen which is a weaker nucleophile than an amino group. Therefore, it is not likely that NADA and NBAD undergo intramolecular cyclization (Fig. 6). Although epinephrine (adrenaline) is $\mathrm{N}$-methylated and is still able to undergo<smiles>NCCc1ccc(O)c(O)c1</smiles><smiles>O=C1C=C2CCNCCC2=CC1=O</smiles>

DAquinone<smiles>[3H][V]</smiles><smiles>Oc1cc2c(cc1O)NCC2</smiles>

leucoDAchrome<smiles>[3H][3H]</smiles><smiles>O=C1C=C2CCN=C2C=C1O</smiles>

DAchrome<smiles>CC(=O)NCCc1ccc(O)c(O)c1</smiles>

Figure 6. Intramolecularcyclization of dopamine (DA) compared to NADA, which cannot undergo this process. The amide nitrogen of NADA and NBAD undergoes resonance and is a poor nucleophile. 
intramolecular cyclization forming adrenochrome, the $\mathrm{N}$ methyl group is not electron withdrawing or bulky compared to an acetyl or $\beta$-alanine group and the amide of NADA and NBAD undergoes resonance. By preventing intramolecular cyclization, this can potentially enhance the crosslinking of their reactive quiones with proteins. 3,4dihydroxyphenylacetaldehyde (DHPAA), formed from oxidation of the amino group of dopamine, is also unable to undergo intramolecular cyclization and may also play an important role in insect cuticle formation (unpublished data).

\section{EVOLUTIONARY PERSPECTIVES}

Generally speaking, melanin polymers provide a protective role in living organisms. The evolvement of tyrosinase is a giant step forward in melanization because tyrosinase (phenoloxidase in insects) catalyzes a two-electron oxidation of melanin precursors and intermediates and 2-electron reduction of oxygen directly to water; thereby reducing the production of semi-quinone radicals and reactive oxygen species. In mammals, the utilization of DCT that catalyzes the isomerization/tautomerization of dopachrome to DHICA further reduces the production of radical species along the melanogenesis pathway because DHICA cannot be easily oxidized by oxygen molecules. Moreover, melanin polymers derived from DHICA seem to be able to better protect mammalian skin from UV irradiation. Therefore, one may consider that evolvement of DCT is another milestone in mammalian melanization. This, however, raises a critical question about the evolvement of DCE that catalyzes a decarboxylative structural rearrangement of dopachrome to $\mathrm{DHI}$ in insects. One might also argue that if $\mathrm{DHI}$ is oxidized more easily, which stimulates the formation of reactive radical species, and the melanin polymers are less resistant against UV irradiation, why has DCE evolved in insects?

In insects, melanization plays diverse roles as compared to mammals. It is involved in cuticle hardening, eggshell hardening, wound healing and melanotic encapsulation of parasites (a part of insect immune responses) (Fig. 7). All these biochemical events must proceed and be completed in a short period of time. For example, during larval development, continued growth requires that insects periodically shed their old cuticle and produce a new one. The newly formed cuticle is soft and elastic, which allows it to stretch and expand to accommodate the increasing body size, but during cuticle regeneration the insect is vulnerable to adverse environmental conditions. Therefore, the soft new cuticle must be hardened or solidified rapidly after insects shed their old cuticle. For wound healing and melanotic parasite encapsulation, the rapidity of the melanization reactions is likely even more critical (Fig. 7). Although the decarboxylative rearrangement of dopachrome to $\mathrm{DHI}$ occurs spontaneously under neutral conditions, this process apparently does not seem to be adequate for insects whose melanization pathway is a major biochemical event relating to cuticle hardening, wound healing, and so forth. Insect DCE facilitates the dopachrome to $\mathrm{DHI}$ pathway thereby tremendously accelerating the insect melanization process.

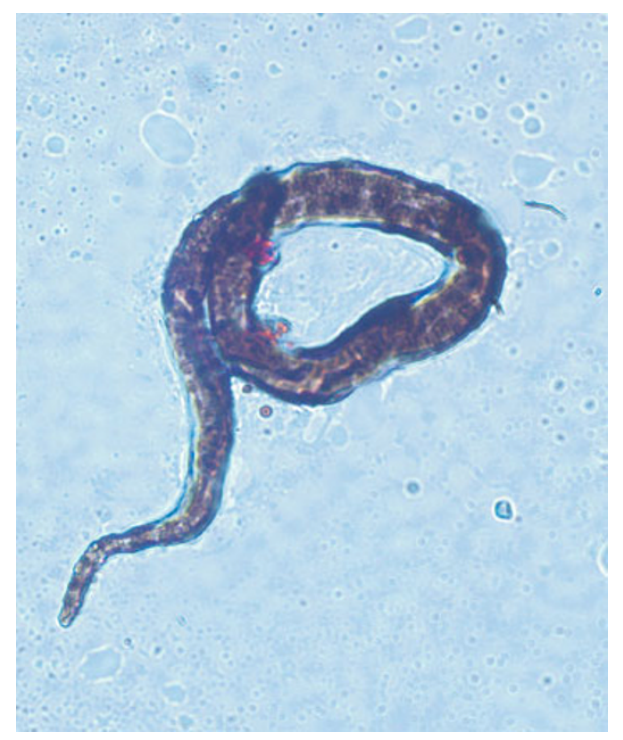

Figure 7. Dirofilaria immitis microfilaraie melanized in Aedes Aegypti.

In mammals, melanization occurs subcellularly (within the melanosome of melanocytes) and is a long-term rather than a momentary event. In humans, although melanization is stimulated considerably upon repetitive exposure to sunlight, it takes days to produce visibly tanned skin. If white human skin turns black upon a few hours of exposure to sunlight, the reactions would have been too rapid and violent to be physiologically beneficial. The life span of human beings is (conservatively) hundreds of times longer than that of many insects. They have much more time to invest into the process of melanogenesis and fine-tuning the pathway to reduce its toxicity. DHICA is much more stable and formation of melanin from DHICA is not as rapid as from DHI because tyrosinase is much less efficient in catalyzing oxidation of DHICA as compared with DHI and DHICA is not oxidized as easily as $\mathrm{DHI}$ by molecular oxygen. We speculate that rather than accelerating melanization, DCT actually slows down the melanization process in mammals, thereby making the process as less toxic as possible. Moreover, melanin polymers produced from DHICA appear more protective against UV irradiation.

The evolution of DCT in mammals and DCE in insects provides an interesting example in terms of ability/capacity of insects and mammals to fine-tune the melanization pathway to best serve their physiological requirements. Production of reactive radicals and reactive oxygen species is a major cause of aging process in humans. The evolvement 
of DCT apparently is a protective adaptation in mammals. For many insects, melanization could be the major biochemical event (at least one of the major biochemical events) in cuticle hardening. Undoubtedly, reactive radicals and reactive oxygen species create oxidative stress to insects, but the speed of melanization is a matter of life and death for them. The life span of most insects is short and their death likely is caused by many other factors than reactive oxygen species. One may speculate that at least for some insects the necessity of rapid melanization apparently overweighs the negative impact of reactive intermediates in the pathway and that the selective pressure for rapid melanization in insects has resulted in the evolvement of DCE.

\section{FUTURE RESEARCH DIRECTIONS}

Direct comparison between the enzymes of melanogenesis and dopa/dopamine metabolism between insects and mammals reveals many interesting evolutionary differences. In regards to melanogenesis, the direct enzymatic regulation in the mammalian system may appear to be more highly controlled. However, the insect pathway is highly evolved as well. Insects contain multiple phenoloxidases and DCE is a member of a yellow gene family with a number of similar proteins. For example, Anopheles gambiae contains at least 9 independent prophenoloxidase sequences (Kim et al., 2005) and Drosophila contains up to 14 yellow family proteins (Drapeau, 2001). Furthermore, insects also contain additional dopa decarboxylase homologs where mammals contain only a single dopa decarboxylase enzyme. Insects must invest a great deal of energy to hardening of their eggs and formation of their cuticle. It is therefore logical that they have evolved a highly complex network of enzymes to assist in these processes. Further characterization of the multitude of yellow family proteins and prophenoloxidase enzymes should be pursued in the future.

Comparison and analysis of the precise distinction between melanization and sclerotization pathways might also reveal some potential research direction especially in regards to differences in polymerization vs. protein crosslinking. It is difficult to separate melanization and sclerotization as two distinct processes and in reality may be impossible to define as separate physiological events. In general, the major distinction that has been made between the intermediates in melanogenesis and sclerotization is the presence or absence of a free amino group, which promotes intramolecular cyclization in the melanin pathway. Although crosslinking precursors NADA and NBAD are generally connected with sclerotization, it is clear that highly reactive quinones arising from melanization pathways also may function to crosslink components in the cuticle. Moreover, our recent data (Vavricka et al., unpublished) suggest that dihydroxyphenylacetaldehyde is also involved in cuticle protein crosslinking. Clearly, the relationship between melanization and sclerotization deserves further investigations.

Additional studies involving the characterization of all the known enzymes involved in melanin biosynthesis, especially the mammalian tyrosinase-related protein family are also needed. Specifically, precise activities of tyrosinase from different species, TRP1 and TRP2 should be clarified. Additional studies at the protein level, especially crystallographic studies, are critical to fully understand the enzymatic and proteomic influence on melanin. A comprehensive understanding of melanin biosynthesis is critical to develop new strategies to deal with melanin related disorders like melanoma skin cancer.

\section{ACKNOWLEDGEMENTS}

This work is supported in part by an NIH grant Al 19769. Chris Vavricka is supported by Chinese Academy of Sciences Fellowship for Young International Scientists (2009Y2BS2) and the National Natural Science Foundation of China (NSFC) Research Fund for International Young Scientists (Grant No. 31050110126). We also thank Matthew T. Aliota from the Christensen Lab at WisconsinMadison Department of Pathobiology for contributing Figure 7. Finally, we are grateful for the assistance of Dr. Qian Han with many of the research projects discussed in this manuscript.

\section{REFERENCES}

Andersen, S.O. (2010). Insect cuticular sclerotization: a review. Insect Biochem Mol Biol 40, 166-178.

Aroca, P., Garcia-Borron, J.C., Solano, F., and Lozano, J.A. (1990). Regulation of mammalian melanogenesis. I: Partial purification and characterization of a dopachrome converting factor: dopachrome tautomerase. Biochim Biophys Acta 1035, 266-275.

Aroca, P., Solano, F., Garcia-Borrón, J.C., and Lozano, J.A. (1991). Specificity of dopachrome tautomerase and inhibition by carboxylated indoles. Considerations on the enzyme active site. Biochem J 277, 393-397.

Aso, Y., Kramer, K.J., Hopkins, T.L., and Whetzel, S.Z. (1984). Properties of tyrosinase and dopa quinone imine conversion factor from pharate pupal cuticle ofManduca sexta L. Insect Biochem 14, 463-472.

Barber, J.I., Townsend, D., Olds, D.P., and King, R.A. (1984). Dopachrome oxidoreductase: a new enzyme in the pigment pathway. J Invest Dermatol 83, 145-149.

Cánovas, F.G., García-Carmona, F., Sánchez, J.V., Pastor, J.L., and Teruel, J.A. (1982). The role of $\mathrm{pH}$ in the melanin biosynthesis pathway. J Biol Chem 257, 8738-8744.

Cardinali, G., Bolasco, G., Aspite, N., Lucania, G., Lotti, L.V., Torrisi, M.R., and Picardo, M. (2008). Melanosome transfer promoted by keratinocyte growth factor in light and dark skin-derived keratinocytes. J Invest Dermatol 128, 558-567.

Cheli, Y., Ohanna, M., Ballotti, R., and Bertolotto, C. (2009). Fifteenyear quest for microphthalmia-associated transcription factor target genes. Pigment Cell Melanoma Res 23, 27-40.

Drapeau, M.D. (2001). The Family of Yellow-Related Drosophila melanogaster Proteins. Biochem Biophys Res Commun 281, 
611-613.

Han, Q., Fang, J., Ding, H., Johnson, J.K., Christensen, B.M., and Li, J. (2002). Identification of Drosophila melanogaster yellow-f and yellow-f2 proteins as dopachrome-conversion enzymes. Biochem $\mathrm{J}$ 368, 333-340.

Hearing, V.J. (1999). Biochemical control of melanogenesis and melanosomal organization. J Investig Dermatol Symp Proc 4, 24-28.

Hearing, V.J., Korner, A.M., and Pawelek, J.M. (1982). New regulators of melanogenesis are associated with purified tyrosinase isozymes. J Invest Dermatol 79, 16-18.

Hernández-Romero, D., Sanchez-Amat, A., and Solano, F. (2006). A tyrosinase with an abnormally high tyrosine hydroxylase/dopa oxidase ratio. FEBS J 273, 257-270.

Hopkins, T.L., Morgan, T.D., Aso, Y., and Kramer, K.J. (1982). N-betaAlanyldopamine: Major Role in Insect Cuticle Tanning. Science 217, 364-366.

Jackson, I.J., Chambers, D.M., Tsukamoto, K., Copeland, N.G., Gilbert, D.J., Jenkins, N.A., and Hearing, V. (1992). A second tyrosinase-related protein, TRP-2, maps to and is mutated at the mouse slaty locus. EMBO J 11, 527-535.

Jara, J.R., Solano, F., and Lozano, J.A. (1988). Assays for mammalian tyrosinase: a comparative study. Pigment Cell Res 1 , 332-339.

Jiang, S., Liu, X.M., Dai, X., Zhou, Q., Lei, T.C., Beermann, F., Wakamatsu, K., and Xu, S.Z. (2010). Regulation of DHICAmediated antioxidation by dopachrome tautomerase: implication for skin photoprotection against UVA radiation. Free Radic Biol Med 48, 1144-1151.

Johnson, J.K., Li, J., and Christensen, B.M. (2001). Cloning and characterization of a dopachrome conversion enzyme from the yellow fever mosquito, Aedes aegypti. Insect Biochem Mol Biol 31 , 1125-1135.

Karlson, P., and Sekeris, C.E. (1962). N acetyl-dopamine as sclerotizing agent of the insect cuticle. Nature 195, 183-184.

Kim, S.R., Yao, R., Han, Q., Christensen, B.M., and Li, J. (2005). Identification and molecular characterization of a prophenoloxidase involved in Aedes aegypti chorion melanization. Insect Mol Biol 14, 185-194.

Korner, A.M., and Gettins, P. (1985). Synthesis in vitro of 5,6dihydroxyindole-2-carboxylic acid by dopachrome conversion factor from Cloudman S91 melanoma cells. J Invest Dermatol 85, 229-231.

Körner, A.M., and Pawelek, J. (1980). Dopachrome conversion: a possible control point in melanin biosynthesis. J Invest Dermatol 75, 192-195.

Lamoreux, M.L., Woolley, C., and Pendergast, P. (1986). Genetic controls over activities of tyrosinase and dopachrome conversion factor in murine melanocytes. Genetics 113, 967-984.

Leonard, L.J., Townsend, D., and King, R.A. (1988). Dopachrome conversion in the eumelanin pathway-product and control. Pigment Cell Res 1, 289-289.

Li, J., Zhao, X., and Christensen, B.M. (1994). Dopachrome conversion activity in Aedes aegypti: significance during melanotic encapsulation of parasites and cuticular tanning. Insect Biochem Mol Biol 24, 1043-1049.

Li, J.S., Ruyl Kim, S., Christensen, B.M., and Li, J. (2005). Purification and primary structural characterization of prophenoloxidases from Aedes aegypti larvae. Insect Biochem Mol Biol 35, 1269-1283.

Li, J.S., Vavricka, C.J., Christensen, B.M., and Li, J. (2007). Proteomic analysis of $\mathrm{N}$-glycosylation in mosquito dopachrome conversion enzyme. Proteomics 7, 2557-2569.

Li, J.Y., and Christensen, B.M. (1994). Effect of pH on the oxidation pathway of dopamine and dopa. J Electroanal Chem 375, 219-231.

Li, J.Y., Zhang, F.J., and Christensen, B.M. (1996). Involvement of lactones in the formation of 6-hydroxydopa and 6hydroxyhydrocaffeic acid during oxidation of dopa and hydrocaffeic acid. J Electroanal Chem 412, 19-29.

Marles, L.K., Peters, E.M., Tobin, D.J., Hibberts, N.A., and Schallreuter, K.U. (2003). Tyrosine hydroxylase isoenzyme I is present in human melanosomes: a possible novel function in pigmentation. Exp Dermatol 12, 61-70.

Matoba, Y., Kumagai, T., Yamamoto, A., Yoshitsu, H., and Sugiyama, M. (2006). Crystallographic evidence that the dinuclear copper center of tyrosinase is flexible during catalysis. J Biol Chem 281, 8981-8990.

Michard, Q., Commo, S., Belaidi, J.P., Alleaume, A.M., Michelet, J.F., Daronnat, E., Eilstein, J., Duche, D., Marrot, L., and Bernard, B.A. (2008a). TRP-2 specifically decreases WM35 cell sensitivity to oxidative stress. Free Radic Biol Med 44, 1023-1031.

Michard, Q., Commo, S., Rocchetti, J., El Houari, F., Alleaume, A. M., Wakamatsu, K., Ito, S., and Bernard, B.A. (2008b). TRP-2 expression protects HEK cells from dopamine- and hydroquinoneinduced toxicity. Free Radic Biol Med 45, 1002-1010.

Nappi, A.J., and Vass, E. (1993). Melanogenesis and the generation of cytotoxic molecules during insect cellular immune reactions. Pigment Cell Res 6, 117-126.

Nappi, A.J., Vass, E., Frey, F., and Carton, Y. (1995). Superoxide anion generation in Drosophila during melanotic encapsulation of parasites. Eur J Cell Biol 68, 450-456.

Olivares, C., Jiménez-Cervantes, C., Lozano, J.A., Solano, F., and García-Borrón, J.C. (2001). The 5,6-dihydroxyindole-2-carboxylic acid (DHICA) oxidase activity of human tyrosinase. Biochem J 354, 131-139.

Pak, B.J., Lee, J., Thai, B.L., Fuchs, S.Y., Shaked, Y., Ronai, Z., Kerbel, R.S., and Ben-David, Y. (2004). Radiation resistance of human melanoma analysed by retroviral insertional mutagenesis reveals a possible role for dopachrome tautomerase. Oncogene 23, 30-38.

Palumbo, A., d'Ischia, M., Misuraca, G., and Prota, G. (1987). Effect of metal ions on the rearrangement of dopachrome. Biochim Biophys Acta 925, 203-209.

Palumbo, A., Solano, F., Misuraca, G., Aroca, P., Garcia Borron, J.C., Lozano, J.A., and Prota, G. (1991a). Comparative action of dopachrome tautomerase and metal ions on the rearrangement of dopachrome. Biochim Biophys Acta 1115, 1-5.

Palumbo, A., Solano, F., Misuraca, G., Aroca, P., Garcia Borron, J.C., Lozano, J.A., and Prota, G. (1991b). Comparative action of dopachrome tautomerase and metal ions on the rearrangement of dopachrome. Biochim Biophys Acta 1115, 1-5.

Pawelek, J.M. (1990). Dopachrome conversion factor functions as an isomerase. Biochem Biophys Res Commun 166, 1328-1333.

Pawelek, J.M., and Lerner, A.B. (1978). 5,6-Dihydroxyindole is a 
melanin precursor showing potent cytotoxicity. Nature 276, 626-628.

Powell, B.J. (2005). 5,6-Dihydroxyindole-2-carboxylic acid: a first principles density functional study. Chem Phys Lett 402, 111-115.

Prota, G. (1992). Melanins and melanogenesis (San Diego, Academic Press).

Townsend, D., Oetting, W.S., Polman, T., and King, R.A. (1992).

Purification and Characterization of Dopachrome-Tautomerase
(Dt). Pigment Cell Research, 32-35.

Vavricka, C.J., Ray, K.W., Christensen, B.M., and Li, J. (2010). Purification and $\mathrm{N}$-glycosylation analysis of melanoma antigen dopachrome tautomerase. Protein J 29, 204-212.

Wang, R.F., Appella, E., Kawakami, Y., Kang, X., and Rosenberg, S. A. (1996). Identification of TRP-2 as a human tumor antigen recognized by cytotoxic $T$ lymphocytes. J Exp Med 184, 2207-2216. 